\title{
Use of Thermophysical Properties to Select and Control Convection During Rapid Solidification of Steel Alloys Using Electromagnetic Levitation on the Space Station
}

\author{
DOUGLAS M. MATSON ${ }^{1,6}$ XIAO XIAO ${ }^{1}{ }^{1}$ JUSTIN E. RODRIGUEZ, ${ }^{1}$ \\ JONGHYUN LEE, ${ }^{2}$ ROBERT W. HYERS, ${ }^{2}$ OLGA SHULESHOVA, ${ }^{3}$ \\ IVAN KABAN,${ }^{3}$ STEPHAN SCHNEIDER,${ }^{4}$ CHRISTIAN KARRASCH, ${ }^{4}$ \\ STEFAN BURGGRAFF ${ }^{4}$ RAINER WUNDERLICH ${ }^{5}$ \\ and HANS-JÖRG FECHT ${ }^{5}$
}

\begin{abstract}
1.-Department of Mechanical Engineering, Tufts University, Medford, MA, USA. 2.-Mechanical and Industrial Engineering Department, University of Massachusetts, Amherst, MA, USA. 3.-Leibniz-Institut für Festerkörper- und Werkstoffforschung (IFW), Dresden, Germany. 4.-Institut für Materialphysik im Weltraum, Deutsches Zentrum für Luft- und Raumfahrt (DLR), Cologne, Germany. 5.-Institut für Mikro-und Nanomaterialen, Universität Ulm, Ulm, Germany. 6.-e-mail: douglas.matson@tufts.edu
\end{abstract}

\begin{abstract}
A major reason to conduct solidification experiments in space is that the unique conditions accessible in reduced-gravity allow investigation of fundamental questions while limiting the influence of sedimentation or buoyancyinduced convection. When processing metallic alloys using containerless electromagnetic levitation, convection may be controlled over a wide range, spanning the laminar-turbulent transition, by proper selection of facility operating conditions. By measuring key thermophysical properties such as density, viscosity, and electrical resistivity on-orbit, the specific sample being processed may be characterized and the results used to update pre-mission magnetohydrodynamic model predictions of induced stirring within the droplet. Thus, convection becomes a controlled experimental parameter that can be applied to an investigation of how stirring influences the metastable-tostable transformation during rapid solidification of $\mathrm{FeCrNi}$ alloys. For these alloys, the incubation or delay time is observed to be a weak function of undercooling and a strong function of applied convection.
\end{abstract}

\section{INTRODUCTION}

Austenitic stainless steels that are specified for use in welding and casting applications are often selected based on a desire to limit hot cracking. A Schaeffler diagram ${ }^{1}$ or DeLong diagram ${ }^{2}$ is used to predict the ferrite content after welding; a Schoefer diagram $^{3}$ is used in a similar manner for casting applications. For the ternary $\mathrm{FeCrNi}$ system, the phase diagram is characterized by a pseudobinary eutectic line running along a composition ratio (CR) $\mathrm{Cr} / \mathrm{Ni}$ ratio of around 1.5 based on solute weight percent ${ }^{4}$ that also corresponds to a decreasing ternary peritectic as the solvent $\mathrm{Fe}$ concentration decreases. The solidification path for a hypoeutectic alloy adjacent to the peritectic line $(1.0<\mathrm{CR}<1.5)$ would involve nucleation of the metastable ferrite (bcc $\delta$-phase) from the liquid with subsequent conversion to stable austenite (fcc $\gamma$-phase) within the metastable mushy zone. This two-step process is known as double recalescence. ${ }^{5}$ Transformation of the skeletal ferrite results in a microstructure resistant to hot cracking ${ }^{6}$ and is known as the ferrite-to-austenite FA-mode behavior. ${ }^{7}$ Phase selection in a macroscopic part would be controlled by the delay, or incubation time, between nucleation events and by the relative growth velocities of the two phases. ${ }^{8}$ It is desirable for this delay to be as long as possible such that most of the casting has experienced double recalescence. This nucleation and growth competition behavior is also observed in a wide range of commercially important alloy systems including $\mathrm{FeNi}^{9}{ }^{9} \mathrm{FeC},{ }^{10} \mathrm{FeCo},{ }^{11} \mathrm{TiAl},{ }^{12,13}$ $\mathrm{CoSi},{ }^{14} \mathrm{TiZrNi}^{15}$ and NdFeB. ${ }^{16}$ 
The results of synchrotron studies ${ }^{10}$ revealed that the close-packed (110) plane of the metastable $\delta$-phase is crystographically coincident with the close-packed (111) plane of the stable $\gamma$-phase in $\mathrm{Fe}-\mathrm{C}$ and that the transformation was massive-like rather than peritectic. Interfacial energies between phases are a strong function of misorientation angle and interface type. ${ }^{17,18}$ The findings from analyses of surface energetics suggest that nucleation occurs within the preexisting metastable $\mathrm{FeCrNi}$ solid ${ }^{19}$ and that nucleation initiates as a peritectic transformation at slow cooling rates, ${ }^{20}$ such as during welding, and as a massive transformation at high cooling rates, such as during casting or rapid solidification processing ${ }^{10}$ in $\mathrm{Fe}-\mathrm{C}$ alloys.

As the thermal driving force that drives secondary recalescence events is independent of stirring or primary undercooling, classic nucleation theory would suggest that the delay is independent of each. ${ }^{19}$ For rapid solidification processing, of particular interest is the observation that the delay time is a weak function of primary undercooling and a strong function of melt stirring, ${ }^{21}$ and this article provides a coherent dataset from a single experimental platform on a single sample to allow new theories to be tested.

To study recalescence phenomena, containerless techniques are used to promote deep undercooling for these highly chemically reactive melts. Two approaches are commonly employed in groundbased research: electrostatic levitation (ESL) and electromagnetic levitation (EML). A key attribute of ESL testing at the NASA Marshall Space Flight Center (MSFC), where levitation is caused by interaction between the sample and a static electric field, is that internal convection is minimized during free cooling such that there is no induced stirring and solidification proceeds on a quiescent sample. In contrast, EML testing is associated with significant induced stirring on a highly turbulent sample caused by the interaction between the sample and the alternating field responsible for overcoming gravity. On the ground, only quiescent or turbulent conditions are accessible. By going to reduced gravity in space, the forces required to position the sample are much smaller, and by using EML, a broad range of convective conditions is accessible. ${ }^{22}$ Predicting fluid flow is accomplished using magnetohydrodynamic (MHD) modeling, which has been validated by comparing liquid recirculation predictions to observations on a levitated CuCo alloy droplet. ${ }^{23}$

Space testing of double recalescence phenomena thus has four key steps. First, thermophysical properties are measured using containerless techniques on the ground to support thermal cycle control modeling activities. Second, MHD modeling is used to design specific run parameters for space experiments that control convection over a wide range of operating conditions. ${ }^{24}$ Third, thermophysical properties are remeasured on orbit to validate and confirm conditions for the particular sample used for space tests. Fourth, recalescence is observed over a wide range of undercooling and fluid flow conditions to define the influence of convection on nucleation phenomena, growth kinetics, phase selection, and metastable phase formation. This article presents a summary of how these steps lead to developing a baseline dataset that may be used to confirm existing or future solidification models.

\section{EXPERIMENTAL}

\section{Ground-Based Experiments at NASA MSFC}

The temperature dependence of key material properties was measured in ground-based ESL tests to facilitate planning of the space processing prior to on-orbit verification for the specific flight EML samples. Electrostatic levitation is accomplished by placing a 40-mg charged spherical sample between a pair of oppositely charged, vertically stacked electrodes at ultra-high vacuum (UHV) to eliminate arcing. A complex control system is used to maintain sample stability, whereas a $200 \mathrm{~W}$ $\mathrm{Nd}$ :YAG laser heating system is used to thermally condition the sample. ESL positioning and heating are thus decoupled.

LED lighting is used to back-light a shadow of the sample into a high-speed camera while temperature is recorded using a one-color pyrometer calibrated in situ at the melt plateau. Density and thermal expansion were measured by superheating a molten sample of known mass and then turning the laser off. Calibrated images taken radially were used to track system volume by assuming axial symmetry. Viscosity and surface tension were measured by melting the sample and then reducing the heating power of the laser to induce an isothermal hold of sufficient duration to excite surface oscillations using the electrostatic field. Mode 2 oscillation amplitude for ESL testing was monitored by measuring the magnitude of the change in dynamic polar diameter after cessation of active excitation. The natural frequency of these oscillations was used to evaluate the surface tension, whereas the oscillation decay was used to evaluate the viscosity. ${ }^{25}$

\section{Ground-Based Experiments at DESY}

In situ time-resolved x-ray diffraction measurements have been performed at the $\mathrm{P} 07^{26}$ highenergy Material Science beamline of the third generation Radiation source PETRA III at the Deutsches Elektron-Synchrotron (DESY), a member of the Helmholtz Association (HGF), outside Hamburg. A total of 1.1-gram samples were processed in a mobile EML developed by IFW-Dresden. For levitation experiments, the sample was placed in a UHV chamber backfilled to 250 mbar with highpurity helium gas after evacuation. Positioning and heating of EML samples was realized through use of 
a water-cooled copper coil powered by a $10-\mathrm{kW}$ generator operating at $280 \mathrm{kHz}$. The sample sits in a potential well generated by the coil magnetic fields. Gravity pulls the sample down into the levitation field causing significant heating and inducing turbulent convection. ${ }^{27}$ Unlike ESL, EML positioning and heating for ground-based testing are thus coupled.

Active cooling was achieved by pumping recirculated inert chamber atmosphere across the sample surface. The sample temperature was measured with a single-color pyrometer, whereas recalescence was imaged using a high-speed digital camera. The structure of the levitated sample was measured in transmission geometry with monochromatic radiation of $121.3 \mathrm{keV}$, or a wavelength of $\lambda=0.1022 \AA$, with a beam size of $500 \times 500 \mu \mathrm{m}^{2}$. The scattered intensity was acquired using a two-dimensional flatpanel Perkin Elmer XRD 1621 x-ray detector configured to provide sufficient counting statistics within the acquisition rate of $5 \mathrm{~Hz}$. The detector was mounted symmetrically around the scattering axis at a $0.8-\mathrm{m}$ sample-to-detector distance. The total scattering intensity is proportional to the scattering vector, $Q$, and was obtained by azimuthal integration of the calibrated and dark current corrected two-dimensional intensity data using the FIT2D software. ${ }^{28,29}$ The alloy used for synchrotron EML processing was selected to obtain incubation delay times compatible with the x-ray detector acquisition rate. For this work, the ternary $\mathrm{Fe}_{72} \mathrm{Cr}_{16} \mathrm{Ni}_{12}$ (wt.\%) alloy samples were produced at the German Space Agency (DLR) by arc-melting of 99.95\% pure elemental feedstock in high-purity $6 \mathrm{~N}$ argon after preconditioning by high-vacuum evacuation.

\section{Magnetohydrodynamic Modeling of Induced Fluid Flow}

Convection inside a EM-levitated molten steel droplet was characterized using the MHD model developed and experimentally validated in the previous research. ${ }^{23}$ Using a finite volume method, this model converts a full Navier-Stokes equations into a system of linear equations, which is then to be solved by a commercial computational fluid dynamics package, ANSYS Fluent. The EM force field was calculated with a subroutine code and implemented into the solver. For turbulence, the renormalization group (RNG) $k-\varepsilon$ model was used as the flow to include the laminar-turbulent transition regime and recirculation fields. ${ }^{30}$ Flow status, flow pattern, and the range of accessible convection were predicted under various test scenarios. The material properties used for the simulations can be found in Ref. 27.

Flow pattern varies as a function of both heating and positioning currents. With the positioning current fixed at $145 \mathrm{~A}$, the heating current was varied from 0 to $150 \mathrm{~A}$. With positioning current alone, two circulations were observed in each hemisphere-one clockwise circulation near the equator and the other in counterclockwise near the pole. As the heating current is increased, the circulation near the pole grows and eventually swallows the other up. This is shown in Fig. 1a. A plot of convection velocity versus heating control voltage can then be developed based on multiple simulation results as shown in Fig. 1b. The control of the positioning and heating currents during space experiments, and the resulting predictions of melt convection, were meticulously planned using the results from the MHD simulations as discussed later.

\section{Rapid Solidification Experiments on the International Space Station}

Undercooling experiments were run over a wide range of melt convection conditions in space using the MSL-EML facility as part of an international collaboration between NASA and ESA. Since processing is conducted in the reduced gravity of space, the magnetic fields needed to contain the sample could be much reduced with a concomitant reduction in induced fluid flow. Sample positioning was controlled using a $150-\mathrm{kHz}$ quadrapole field, and heating was accomplished by overlaying an independent $350-\mathrm{kHz}$ dipole field onto the same SUPOS coil system-an acronym for superposition. Thus, in space, EML positioning and heating were again decoupled.

Two types of experiments could be run. ${ }^{31}$ First, thermophysical properties were spot-checked to define how the specific flight sample behaves. The top-view axial camera and pyrometer (ACP) simultaneously recorded temperature and video images of the sample surface for facility health monitoring. Onboard software was subsequently used to trigger the acquisition of density or viscosity video images; either dynamic equatorial diameter or integrated sample area could be used for analysis. Second, high-speed imaging was used to study solidification phenomena. The side-view radial camera (RAD) ran at $30 \mathrm{kHz}$ with a 0.3 -s pre-trigger period programmed prior to recalescence. Note that property measurement and solidification studies could be conducted during the same thermal cycle through independent analyses of the ACP and RAD images.

These two types of experiments are shown in Fig. 2. In the top part of the image, the timetemperature profile is displayed in blue on the graph. Green shows a trace of the positioner, and red shows a trace of the heater control voltage setting. During free-cooling, two pulses from the dipole heating field were used to excite mode 2 surface oscillations by squeezing-in radially at the equator. Sample area as a function of time is plotted in the lower-left box in Fig. 2a from analysis of the ACP images. Temperature was continuously changing throughout the decay process, and thus, 


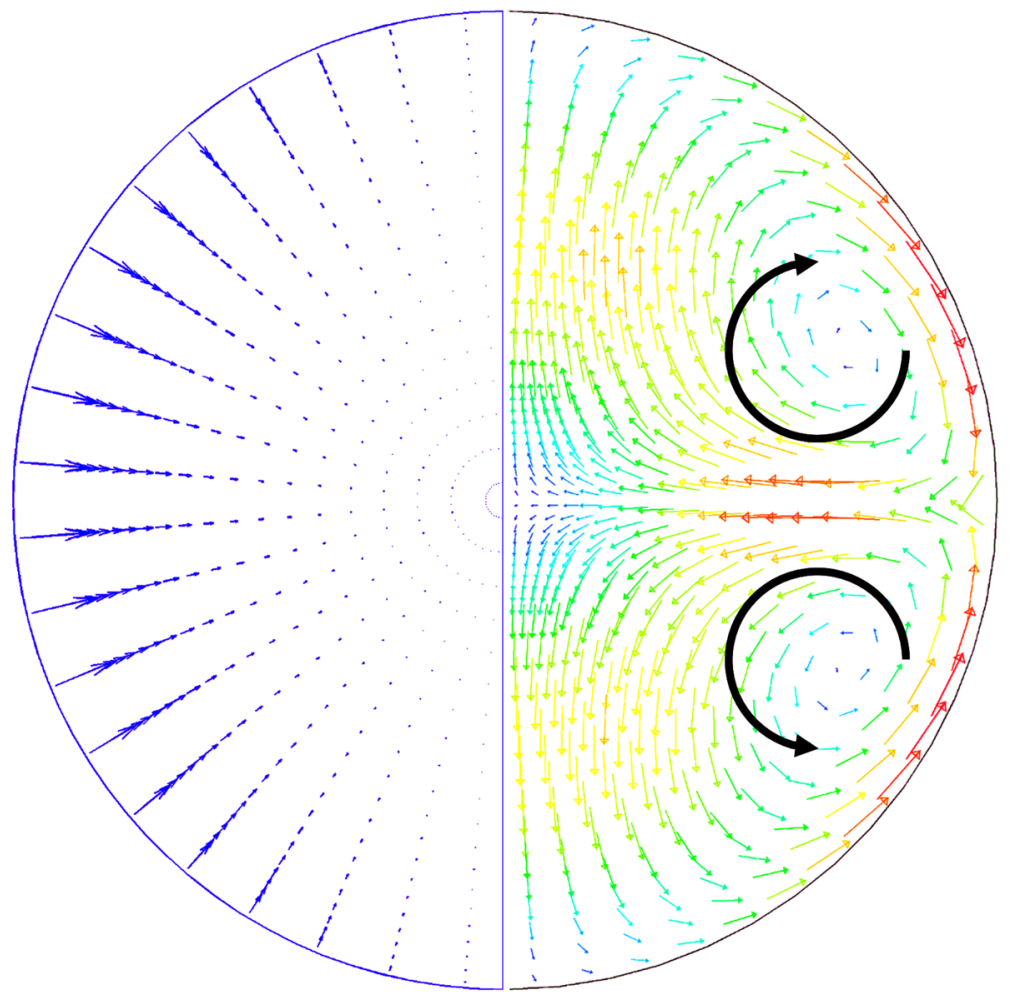

(a)

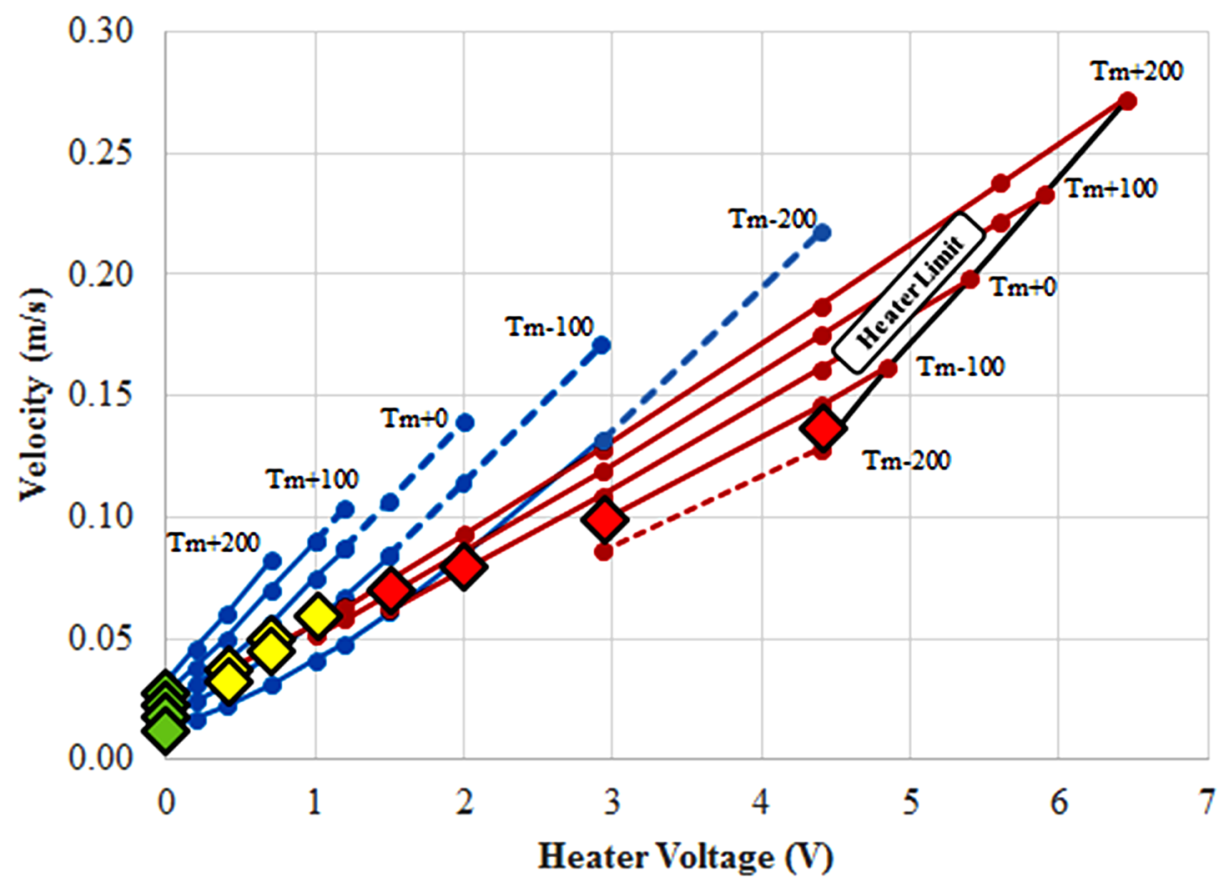

(b)

Fig. 1. Control of convection: (a) MHD model result and (b) range accessible in space tests. 
multiple measurements over small time periods, and the associated small temperature ranges, could be accomplished throughout a single run.

Solidification behavior is highlighted in the lowerright box in Fig. $2 b$, and a representative RAD image showing double recalescence is displayed. The undercooled liquid is self-illuminating, and with the selection of appropriate aperture settings appears as a dark gray against a black background. Near the center of the sample, the light gray metastable phase has nucleated and grows into the undercooled liquid. The stable phase appears as a brighter (and, thus, hotter) region at the center of this metastable region.

The nominal 1.1-g spherical samples were produced at the University of Ulm by vacuum casting of ingot sections taken from a master-alloy produced from $4 \mathrm{~N}$ elemental feedstock. For this work, the ternary $\mathrm{Fe}_{60} \mathrm{Cr}_{20} \mathrm{Ni}_{20}$ (wt.\%) alloy was selected to observe fast transformation kinetics compatible with the high-speed camera acquisition rate. Tests were conducted at $35,000 \mathrm{~Pa}$ (350 millibar) backpressure to limit any composition shift caused by the potential for preferential evaporation of chromium from the alloy. In space, most tests were pressurized using helium, although a limited set was tested using argon so that any influence on solidification phenomena introduced by changing the cooling rate could be investigated independently from any influence from convection.

\section{RESULTS AND DISCUSSION}

\section{Convection Control}

To plan the space experiments, thermophysical properties were measured on the ground and the results used to conduct a broad range of MHD calculations on possible run configurations. From this broad set of results, key parameters were identified and simulations were focused to generate the desired operational conditions for the flight experiment. On-orbit, property evaluations were accomplished in situ to confirm the specific properties of the space sample [Orbit], and MHD models were reevaluated. Figure 1a shows a typical MHD result for a given configuration of sample size, temperature, heater control voltage setting, and positioner control voltage setting. On the left side of the spherical sample displayed in the figure, the force distribution is shown corresponding to a condition where no excitation pulse is being applied. On the right, flow patterns show the formation of

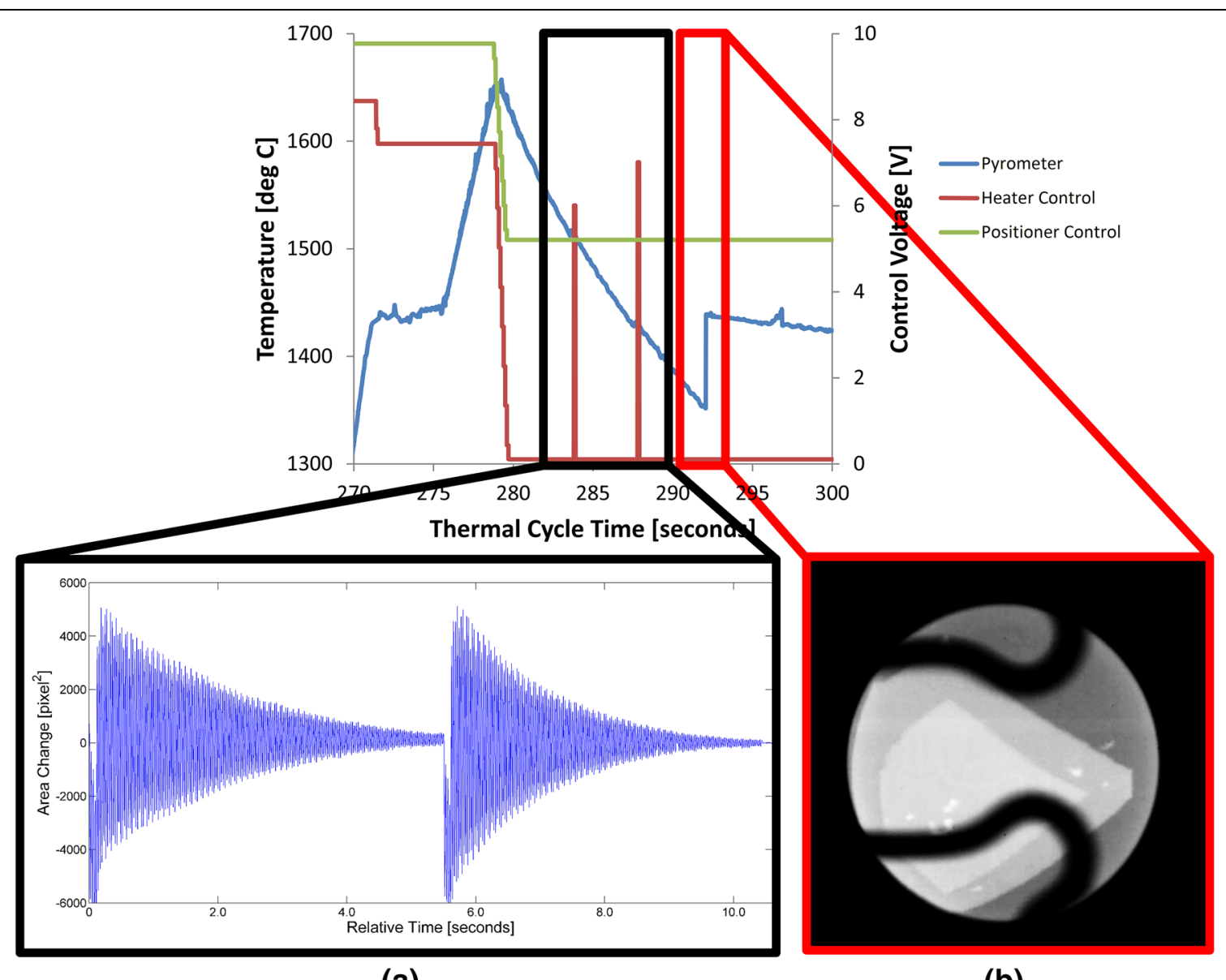

(a)

(b)

Fig. 2. Types of space testing: (a) thermophysical property evaluation and (b) rapid solidification. 


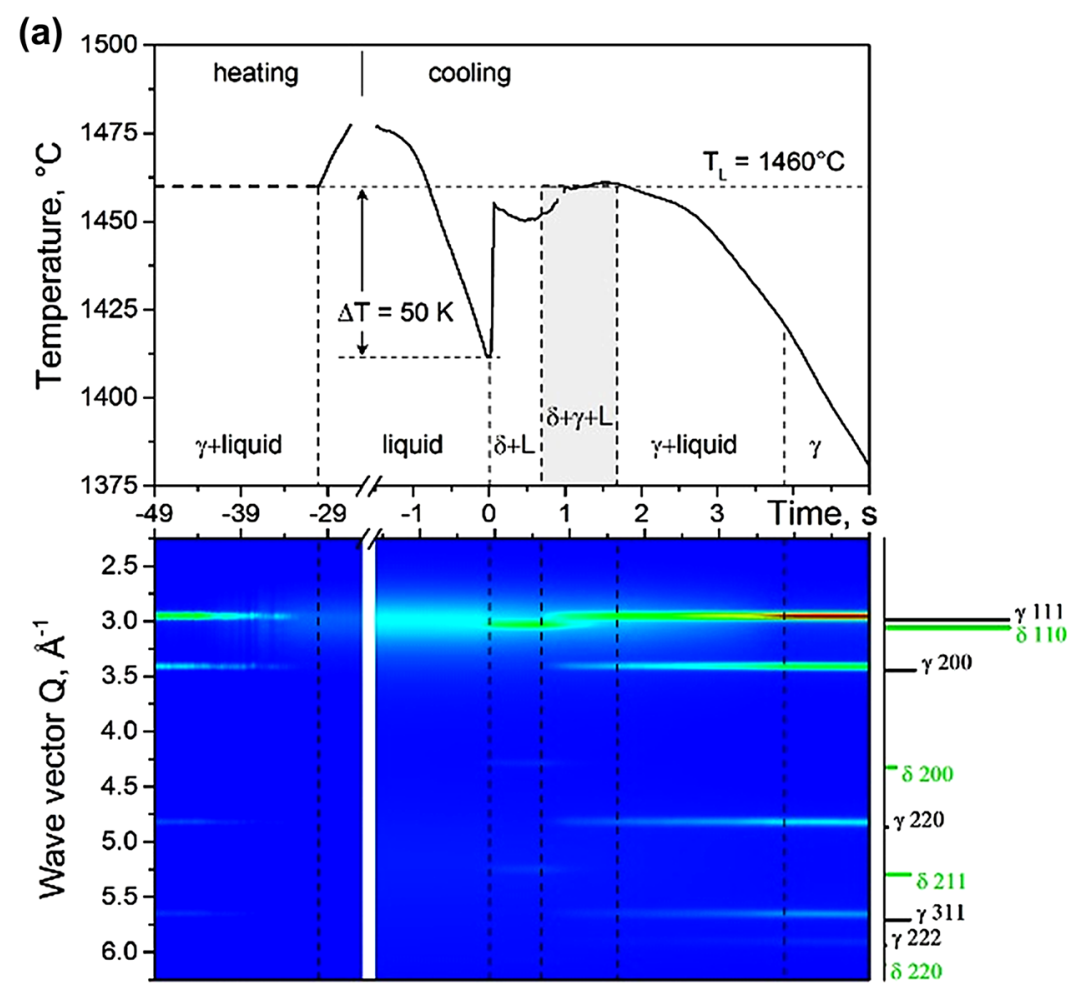

(b)

Fe-12Ni-16Cr solidification at $\Delta \mathrm{T}=50 \mathrm{~K}$

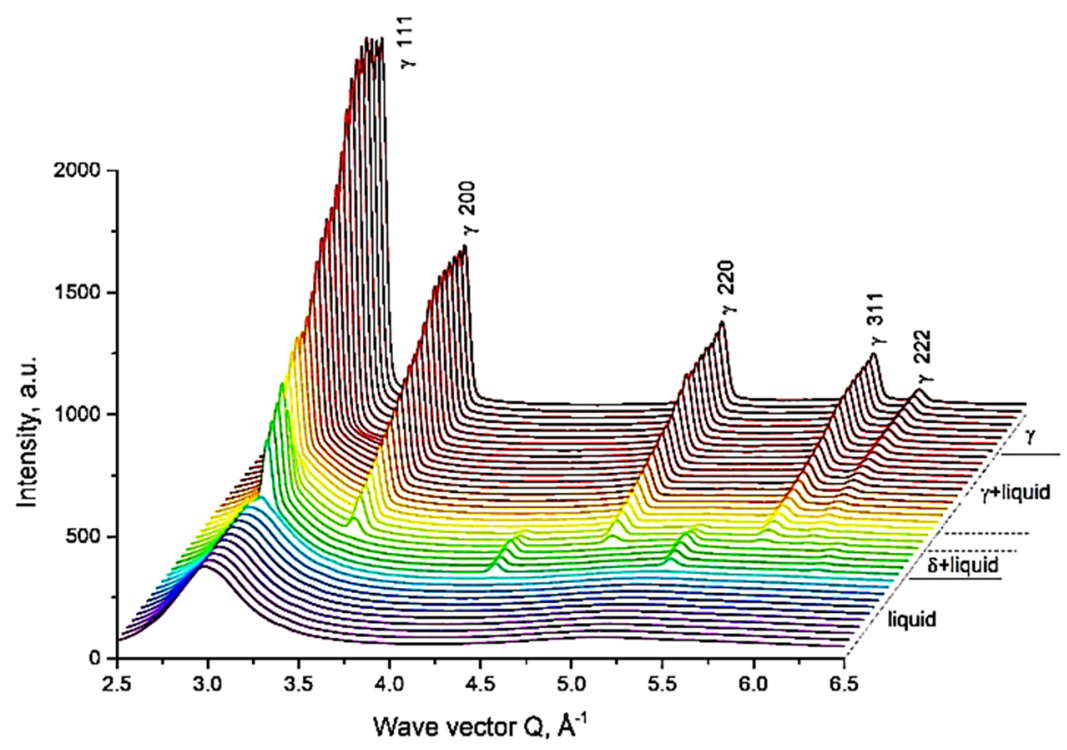

Fig. 3. DESY Synchrotron evaluation during steel double recalescence: (a) wave vector plot and (b) intensity plot.

two opposing toroidal loops-one in the upper and one in the lower hemisphere. The graph in Fig. 1b shows the range of actual run conditions that were accomplished during the space testing. Note that the recirculation loop velocities are predicted to be laminar at $0.02 \mathrm{~m} / \mathrm{s}$ and turbulent at $0.20 \mathrm{~m} / \mathrm{s}$, respectively, for control voltage settings from 0 to $5 \mathrm{~V}$. The green region shows the convection velocity at superheated temperatures for both laminar and turbulent regimes. The blue and red regions represent undercooled temperatures for laminar and turbulent regimes, respectively. Convection velocity increases as the temperature is elevated. The maximum convection velocity increases as more heating control voltage is added to the coil. The trimmed region (bold solid black line) on the curves represents the limit to convection based on overheating of the undercooled sample. Figure $1 \mathrm{~b}$ shows 
the heating control voltages used in the space experiments with the resulting prediction of recirculation velocities on-orbit; green symbols indicate laminar conditions, red indicate fully developed turbulent conditions, and yellow indicate the transition between these extremes. Liquid shear may also be evaluated from the MHD simulation results. For the conditions run, shear was approximately linearly dependent on the recirculation velocity and the predicted values for each test were tabulated for use in predicting the damage parameter for delay time model evaluations.

\section{Solidification Path}

Time-resolved $\mathrm{x}$-ray diffraction investigations on weld solidification show primary ferrite (FA-mode) or primary austenite (AF-mode) with subsequent melting of the metastable primary phase; the solidification path depends on both the weld speed and composition. $^{32}$ Rapid solidification can be much different. ${ }^{33}$ Figure 3 shows DESY synchrotron processing such that the equilibrium $\gamma$-phase fcc-peaks disappear once the solid is molten. On solidification, primary $\delta$-ferrite forms and then is subsequently melted during the formation of austenite such that only austenite then remains (FA-mode); the bccpeaks have a lifetime of about $1 \mathrm{~s}$. This progression is also shown as an intensity plot in the second part of the figure. Note that low-temperature transformations are not documented here as this article concentrates on rapid solidification of the neareutectic stainless steel alloy family.

\section{Transformation Delay}

The high-speed video was used to identify when and where the two recalescence events initiated to evaluate the delay time between them. Undercooled liquid is dark gray, metastable ferrite mid-gray, and stable austenite light-gray on a black background. Figure 4 shows two representative images from the video recordings of the space tests. The first test was run with minimal stirring $(0.02 \mathrm{~m} / \mathrm{s})$. Under laminar conditions, the delay between primary and secondary recalescence events was long giving enough time for the primary metastable phase to grow across the entire surface of the droplet before nucleation of the stable phase. The second sample was run with significant stirring $(0.12 \mathrm{~m} / \mathrm{s})$. Under turbulent conditions, the delay was short and the metastable phase had not grown far into the undercooled liquid before nucleation of the second phase occurred. Delay results were independent of test atmosphere where with minimal stirring cooling rates in argon and helium were 20 and $50 \mathrm{~K} / \mathrm{s}$, respectively.

The second part of Fig. 4 shows a graph of delay time as a function of undercooling for various run scenarios. The orange circles represent ESL ground-testing with no induced stirring; black squares represent EML ground-testing with turbulent flow. The space tests are shown with diamonds; red represents fully turbulent results where shear is also on the order of the groundbased results, yellow represents transitional conditions, and green represents laminar flow at the lowest induced flow conditions possible in reduced gravity. The solid line shows how ESL behavior deviates significantly from the range of spaceaccessible conditions shown by the dotted lines. These results conclusively show that convection significantly decreases the delay time. Turbulent space and turbulent ground EML tests show a similar behavior and laminar space results approach but do not achieve the delay behavior of quiescent ground ESL. (a)

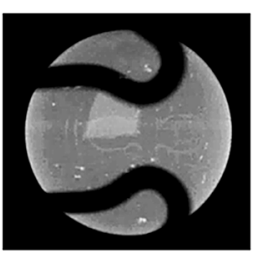

(b)

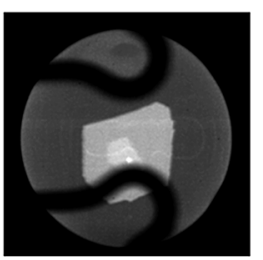

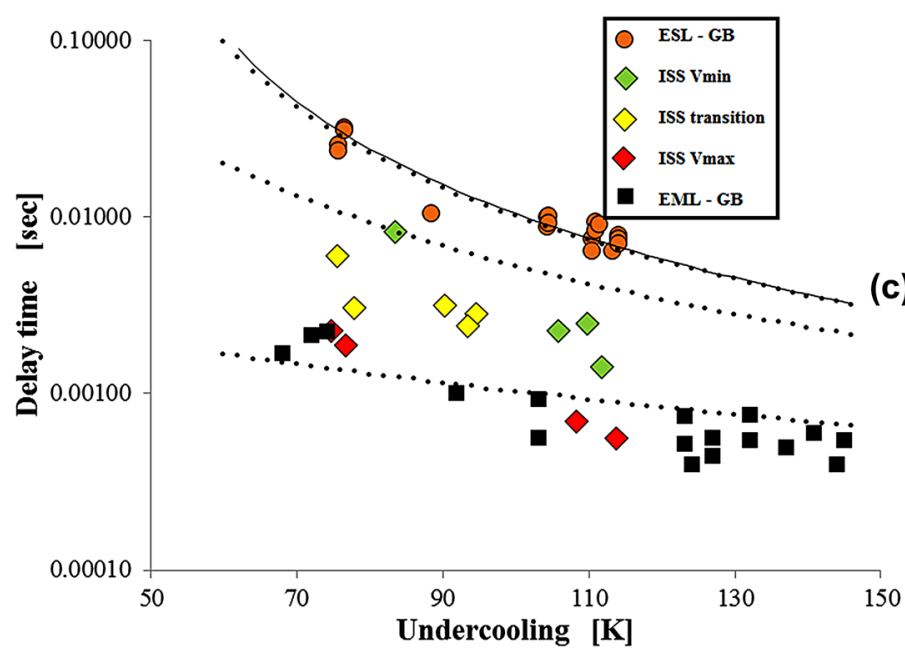

Undercooling $[\mathbf{K}]$

Fig. 4. Influence of convection on incubation time between nucleation of metastable phase and subsequent transformation to the stable phase: (a) low stirring = long time, (b) high stirring = short time, and (c) relationship between delay and undercooling as a function of experimental conditions. 


\section{CONCLUSION}

Thermophysical properties can be measured on the ground before a space mission and can be used to predict the range of potential thermal cycle run conditions that may be achieved using MHD techniques. Remeasurement on-orbit provides validation of actual run conditions. In space, a wide range of stirring conditions are possible-from laminar to turbulent-allowing investigation of phase selection behavior. No difference was seen among vacuum, $\mathrm{Ar}$, and $\mathrm{He}$ environments, indicating that the cooling rate is not a factor in determination of the delay between stable and metastable phase formation. Space testing results support the prediction that both undercooling and melt stirring will reduce the observed delay.

\section{ACKNOWLEDGEMENTS}

Funding supporting this work was provided by NASA under grant number NNX16AB59G and NNX16AB40G, by DLR under contract 50WM1170 at Ulm University and PARMAG contract 50WM1546 at IFW Dresden, and by the ESA MAP Project Thermoprop AO-99-022 and AO-2009-1020 contract number 4200014306. The authors would also like to thank Olof Gutowski and Dr. Uta Rütt at DESY for assistance in using the high-energy materials science beamline P07 and ESL staff Mike SanSoucie, Trudy Allen, and Glenn Fountain at NASA Marshall Space Flight Center in Huntsville, $A L$, for ground-based thermophysical property measurement experiment support.

\section{REFERENCES}

1. A.L. Schaeffler, Metal Prog. 56, 680 (1949).

2. W.T. DeLong, G.A. Ostrom, and E.R. Szumachowski, Weld. J. Res. Suppl. 35, 526 (1956).

3. ASTM A800-1, Standard Practice for Steel Casting, Austenitic Alloy, Estimating Ferrite Content (West Conshohocken: ASTM International, 2024), p. 1.

4. N. Suutala, Metall. Trans. A 14A, 191 (1983).

5. T. Koseki and M.C. Flemings, Metall. Mater. Trans. A 26A, 2991 (1995)

6. J.A. Brooks, J.C. Williams, and A.W. Thompson, Metall. Trans. A 14A, 1271 (1983).

7. T. Koseki and M.C. Flemings, Metall. Mater. Trans. A 27A, 3236 (1996).

8. D.M. Matson, Materials in Space-Science, Technology, and Exploration, MRS Symposium Proceedings, vol. 551, eds. by A.F. Hepp, J.M. Prahl, T.G. Keith, S.G. Bailey, J.R. Fowler (Warrendale: Materials Research Society, 1999), p. 227.
9. K. Eckler, F. Gärtner, H. Assadi, A.F. Norman, A.L. Greer, and D.M. Herlach, Mater. Sci. Eng. A A226-A228, 410 (1997).

10. H. Yasuda, T. Nagira, M. Yoshiya, A. Sugiyama, N Nakatsuka, M. Kiire, M. Uesugi, K. Uesugi, K. Umetani, and K. Kajiwara, IOP Conf. Ser. Mater. Sci. Eng. 33, 012036 (2012).

11. J.E. Rodriguez, C. Kreischer, T. Volkmann, and D.M. Matson, Acta Mater. 122, 431 (2017).

12. O. Shuleshova, D. Holland-Moritz, W. Löser, A. Voss, H. Hartmann, U. Hecht, V.T. Witusiewicz, D.M. Herlach, and B. Büchner, Acta Metall. 58, 2408 (2010).

13. D. Veeraraghavan, P. Wang, and V.K. Vasudevan, Acta Metall. 47, 3313 (1999).

14. Y.K. Zhang, J. Gao, M. Kolbe, S. Klein, C. Yang, H. Yasuda, D.M. Herlach, and C.-A. Gandin, Acta Mater. 61, 4861 (2013).

15. G.W. Lee, A.K. Gangopadhyay, R.W. Hyers, T.J. Rathz, J.R. Rogers, D.S. Robinson, A.I. Goldman, and K.F. Kelton, Phys. Rev. B 77, 184102 (2008).

16. J. Strohmenger, T. Volkmann, J. Gao, and D.M. Herlach, Mater. Sci. Eng. A 413-414, 263 (2005).

17. M. Yoshiya, K. Nakajima, M. Watanabe, N. Ueshima, T. Nagira, and H. Yasuda, Metall. Mater. Trans. 56, 1461 (2015).

18. M. Yoshiya, M. Watanabe, I.K. Nakajima, N. Ueshima, K. Hashimoto, T. Nagira, and H. Yasuda, Metall. Mater. Trans. 56, 1467 (2015).

19. D.M. Matson, Solidification of Containerless Undercooled Melts, ed. D.M. Herlach and D.M. Matson (Weinheim: Wiley, 2012), p. 213.

20. B.K. Dhindaw, T. Antonsson, J. Tinoco, and H. Fredriksson, Metall. Mater. Trans. A 35A, 2869 (2004).

21. A.B. Hanlon, D.M. Matson, and R.W. Hyers, Philos. Mag. Lett. 86, 165 (2006).

22. J. Lee, X. Xiao, D.M. Matson, and R.W. Hyers, Metall. Mater. Trans. B 46B, 199 (2015).

23. J. Lee, D.M. Matson, S. Binder, M. Kolbe, D. Herlach, and R.W. Hyers, Metall. Mater. Trans. B 45B, 1018 (2014).

24. A.B. Hanlon, D.M. Matson, and R.W. Hyers, Ann. NY Acad. Sci. 1077, 33 (2006)

25. D.M. Matson, M. Watanabe, G. Pottlacher, G.W. Lee, and H.-J. Fecht, Int. J. Microgr. Sci. Appl. 33, 330301 (2016).

26. N. Schell, A. King, F. Beckmann, T. Fischer, M. Müller, and A. Schreyer, Mater. Sci. Forum 772, 57 (2014).

27. R.W. Hyers, D.M. Matson, K.F. Kelton, and J.R. Rogers, Ann. NY Acad. Sci. 1027, 474 (2004).

28. A.P. Hammersley, S.O. Svensson, M. Hanfland, A.N. Fitch, and D. Hausermann, High Pressure Res. 14, 235 (1996).

29. A.P. Hammersley, ESRF Internal Report, ESRF98HA01T, FIT2D V9.129 Reference Manual V3.1 (1998).

30. S. Berry, R.W. Hyers, B. Abedian, and L.M. Racz, Metall. Mater. Trans. B 31B, 178 (2000).

31. D.M. Matson, X. Xiao, J. Rodriguez, and R.K. Wunderlich, Int. J. Microgr. Sci. Appl. 33, 330206 (2016).

32. S.S. Babu, J.W. Elmer, J.M. Vitek, and S.A. David, Acto Mater. 50, 4763 (2002)

33. H. Inoue and T. Koseki, Acta Mater. 124, 430 (2017). 